\title{
On Homeomorphism Between Euclidean Subspace and Conformally Euclidean Manifold
}

\author{
Ashot S. Gevorkyan ${ }^{1,2}$, Alek A. Aleksanyan ${ }^{1}$ and Suren B. Alaverdyan ${ }^{1}$ \\ ${ }^{1}$ Institute for Informatics and Automation Problems of NAS RA \\ ${ }^{2}$ Institute of Chemical Physics after A. B. Nalbandyan of NAS RA \\ e-mail: gashot@ipia.sci.am
}

\begin{abstract}
The article presents the proof of the homeomorphism between Euclidean subspace $\mathbb{E}^{6}$ of the classical three-body system and $6 D$ Riemannian manifold $\mathcal{M}$, which allows reducing the dynamical problem to the system of the 6th-order.
\end{abstract}

Keywords: System of underdetermined algebraic equations, Orientated $3 D$ Riemannian manifold, Topology of $3 D$ manifolds.

\section{Introduction}

As is known, the time evolution of the classical system is uniquely determined by the Hamilton equations and is usually reduced to a system of ordinary differential equations of the second order. Integrating this system of a differential equation means finding all possible functions of one variable " $t$ " (time), which, when substituted into equations, turns them into an identity. In the case of dynamical systems, as a rule, the system of equations cannot be fully integrated, since the number of integrals of motion often is less than the number of degrees of freedom.

In the series of works [1]-[5], using the example of the classical three-body problem, it was shown that the use of Riemannian geometry makes it possible to reveal new hidden symmetries of a dynamical system, which makes the integration of the problem more completel.

In this paper we examine the question of homeomorphism between $6 D$ Euclidean subspace $\mathbb{E}^{6}$ and $6 D$ manifold $\mathcal{M}$. In particular, the question of the decomposition of a manifold in the form $\mathcal{M}: \Leftrightarrow \mathcal{M}^{(3)} \times \mathcal{S}_{M_{i}}^{3}$ is proved, where $\mathcal{M}^{(3)}$ denotes the sum of 84 oriented in $9 D$ Euclidean space $3 D$ manifolds and $\mathcal{S}_{M_{i}}^{3}$ is the group symmetry $S O(3)$ at the given point $M_{i} \in \mathcal{M}^{(3)}$. 


\section{On Homeomorphism Between the Euclidean Subspace and the Confor- mally Euclidean Manifold}

Proposition 1: Let $\mathbb{E}^{6}$ be a Euclidean subspace with metric $\gamma_{\mu \nu}(\{\rho\})$, on which an orthogonal coordinate system is given:

$$
\rho_{1}, \ldots, \rho_{6}=\{\rho\}=\overline{\rho_{1}, \rho_{6}} \in \mathbb{E}^{6}
$$

and, respectively, $\mathcal{M}$ is a conformally Euclidean manifold, which is determined by the metric tensor $g_{\alpha \beta}(\{x\})$ and the local coordinate system $\{x\}$ :

$$
g_{\alpha \beta}(\{x\})=g(\{\bar{x}\}) \delta_{\alpha \beta}, \quad\{x\}=x^{1}, \ldots, x^{6}, \quad\{\bar{x}\}=\overline{x^{1}, x^{3}}, \quad \alpha, \beta=\overline{1,6},
$$

where $g(\{\bar{x}\})>0$ is a smooth function belonging to the class $\mathbb{C}^{1}\left(\mathbb{R}^{6}\right)$, then the Euclidean subspace $\mathbb{E}^{6}$ is homeomorphic to the manifold $\mathcal{M}$.

Proof. Let us consider a linear infinitesimal element " $d s "$ in both coordinate systems $\{\rho\} \in \mathbb{E}^{6}$ and $\{x\} \in \mathcal{M}$. Equating them, we can write:

$$
(d s)^{2}=\gamma^{\alpha \beta}(\{\rho\}) \mathrm{d} \rho_{\alpha} \mathrm{d} \rho_{\beta}=\mathrm{g}_{\mu \nu}(\{\overline{\mathrm{x}}\}) \mathrm{dx}^{\mu} \mathrm{dx}^{\nu}, \quad \alpha, \beta, \mu, \nu=\overline{1,6},
$$

from which one can obtain the following system of algebraic equations:

$$
\gamma^{\alpha \beta}(\{\rho\}) \rho_{\alpha, \mu} \rho_{\beta, \nu}=\mathrm{g}_{\mu \nu}(\{\overline{\mathrm{x}}\})=\mathrm{g}(\{\overline{\mathrm{x}}\}) \delta_{\mu \nu},
$$

where it is necessary to prove that the coefficients $\rho_{\alpha, \mu}(\{\mathrm{x}\})=\partial \rho_{\alpha} / \partial \mathrm{x}^{\mu}$ make sense of derivatives. In this regard, we must prove that the function $\rho_{\alpha}(\{x\})$ is twice differentiable and continuous in its domain of definition and, in addition, satisfy the symmetry condition:

$$
\rho_{\alpha, \mu \nu}(\{\mathrm{x}\})=\rho_{\alpha, \nu \mu}(\{\mathrm{x}\}), \quad \forall \quad \mu, \nu=\overline{1,6},
$$

(Schwartz's theorem on the symmetry of second derivatives). Recall that the set of coefficients $\rho_{\alpha, \mu}(\{\mathrm{x}\})$ allows us to perform coordinate transformations $\{\rho\} \mapsto\{x\}$, which we shall call direct transformations.

Similarly, from (3), one can obtain a system of algebraic equations defining inverse transformations:

$$
\gamma_{\alpha \beta}(\{\rho\}) g^{-1}(\{\bar{x}\})=x_{, \alpha}^{\mu} x_{, \beta}^{\nu} \delta_{\mu \nu}
$$

where $x^{\mu}{ }_{\alpha}(\{\rho\})=\partial x^{\mu} / \partial \rho^{\alpha}$ and $\gamma_{\alpha \beta}(\{\rho\})=\gamma_{\alpha \bar{\alpha}}(\{\rho\}) \gamma_{\beta \bar{\beta}}(\{\rho\}) \gamma^{\bar{\alpha} \bar{\beta}}(\{\rho\})$.

At first we consider the system of equations (4), which is related to direct coordinate transformations. It is easy to see that the system of algebraic equations (4) is underdetermined with respect to the variables $\rho_{\alpha, \mu}(\{\mathrm{x}\})$, since it consists of 21 equations, while the number of unknown variables is 36 . Obviously, when these equations are compatible, then the system of equations (4) has an infinite number of real and complex solutions. Note that for the classical three-body problem, the real solutions of the system (4) are important, which form a 15-dimensional manifold. Since the system of equations (6) is still defined in a rather arbitrary way we can impose additional conditions on it in order to find the minimal dimension of the manifold allowing a separation of the base $\mathcal{M}^{(3)}$ from the layer $\cup_{i} \mathcal{S}_{M_{i}}^{3}$. 
Let us make new notations:

$$
\alpha_{\mu}=\rho_{1, \mu}, \quad \beta_{\mu}=\rho_{2, \mu}, \quad \zeta_{\mu}=\rho_{3, \mu}, \quad u_{\mu}=\rho_{4, \mu}, \quad v_{\mu}=\rho_{5, \mu}, \quad w_{\mu}=\rho_{6, \mu} .
$$

We also require that the following additional conditions be met:

$$
\begin{aligned}
& \alpha_{4}=\alpha_{5}=\alpha_{6}=0, \quad \beta_{4}=\beta_{5}=\beta_{6}=0, \quad \zeta_{4}=\zeta_{5}=\zeta_{6}=0 \\
& u_{1}=u_{2}=u_{3}=0, \quad v_{1}=v_{2}=v_{3}=0,
\end{aligned}
$$

Using (7) and conditions (8) from the equation (4) we can obtain two independent systems of algebraic equations:

$$
\begin{array}{rlrl}
\alpha_{1}^{2}+\beta_{1}^{2}+\gamma^{33} \zeta_{1}^{2} & =\breve{g}(\{\bar{\rho}\}), & \alpha_{1} \alpha_{2}+\beta_{1} \beta_{2}+\gamma^{33} \zeta_{1} \zeta_{2}=0, \\
\alpha_{2}^{2}+\beta_{2}^{2}+\gamma^{33} \zeta_{2}^{2}=\breve{g}(\{\bar{\rho}\}), & \alpha_{1} \alpha_{3}+\beta_{1} \beta_{3}+\gamma^{33} \zeta_{1} \zeta_{3}=0, \\
\alpha_{3}^{2}+\beta_{3}^{2}+\gamma^{33} \zeta_{3}^{2}=\breve{g}(\{\bar{\rho}\}), & \alpha_{2} \alpha_{3}+\beta_{2} \beta_{3}+\gamma^{33} \zeta_{2} \zeta_{3}=0,
\end{array}
$$

(recall that at obtaining (9) it is assumed that $\gamma^{11}=\gamma^{22}=1$ ) and, correspondingly:

$$
\begin{array}{r}
\gamma^{44} u_{4}^{2}+\gamma^{55} v_{4}^{2}+\gamma^{66} w_{4}^{2}+2\left(\gamma^{45} u_{4} v_{4}+\gamma^{46} u_{4} w_{4}+\gamma^{56} v_{4} w_{4}\right)=\breve{g}(\{\bar{\rho}\}), \\
\gamma^{44} u_{5}^{2}+\gamma^{55} v_{5}^{2}+\gamma^{66} w_{5}^{2}+2\left(\gamma^{45} u_{5} v_{5}+\gamma^{46} u_{5} w_{5}+\gamma^{56} v_{5} w_{5}\right)=\breve{g}(\{\bar{\rho}\}), \\
\gamma^{44} u_{6}^{2}+\gamma^{55} v_{6}^{2}+\gamma^{66} w_{6}^{2}+2\left(\gamma^{45} u_{6} v_{6}+\gamma^{46} u_{6} w_{6}+\gamma^{56} v_{6} w_{6}\right)=\breve{g}(\{\bar{\rho}\}), \\
a_{4} u_{4}+a_{5} v_{4}+a_{6} w_{4}=0, \\
b_{4} u_{5}+b_{5} v_{5}+b_{6} w_{5}=0, \\
c_{4} u_{6}+c_{5} v_{6}+c_{6} w_{6}=0 .
\end{array}
$$

In equations (10), the following notations are made:

$$
a_{i}=\gamma^{i 4} u_{5}+\gamma^{i 5} v_{5}+\gamma^{i 6} w_{5}, \quad b_{j}=\gamma^{j 4} u_{6}+\gamma^{j 5} v_{6}+\gamma^{j 6} w_{6}, \quad c_{k}=\gamma^{k 4} u_{4}+\gamma^{k 5} v_{4}+\gamma^{k 6} w_{4},
$$

where $i, j, k=\overline{4,6}$.

It should be noted that the solutions of algebraic systems (9) and (10) form two different $3 D$ manifolds $\mathfrak{S}^{(3)}$ and $\mathfrak{R}^{(3)}$, respectively. The manifold $\mathfrak{S}^{(3)}$ is in a one-to-one mapping on the one hand with the subspace $\mathbb{E}^{3} \ni\{\bar{\rho}\}$ (where $\mathbb{E}^{3} \subset \mathbb{E}^{6}$ the internal space in the hyperspherical coordinate system), and on the other hand with the manifold $\mathcal{M}^{(3)}$ (see Fig. $1)$. Note that this statement follows from the fact that all points of the manifold $\mathcal{M}^{(3)}$ and the subspace $\mathbb{E}^{3}$, are pairwise connected through the corresponding derivatives (see (4)), which, as unknown variables, enter the algebraic equations (9), and, in addition, as shown there exist also inverse coordinate transformations (see Appendix).

Now we prove the continuity of these mappings.

Recall that the unknowns in the equations (9), are in fact functions of coordinates $\{\bar{\rho}\}$. Performing a shift of coordinates $\{\bar{\rho}\} \rightarrow\{\bar{\rho}\}+\{\delta \bar{\rho}\}$ in (9), we get the following system of equations:

$$
\begin{aligned}
\bar{\alpha}_{1}^{2}+\bar{\beta}_{1}^{2}+\bar{\gamma}^{33} \bar{\zeta}_{1}^{2}=\bar{g}(\{\bar{\rho}\}), & \bar{\alpha}_{1} \bar{\alpha}_{2}+\bar{\beta}_{1} \bar{\beta}_{2}+\bar{\gamma}^{33} \bar{\zeta}_{1} \bar{\zeta}_{2}=0, \\
\bar{\alpha}_{2}^{2}+\bar{\beta}_{2}^{2}+\bar{\gamma}^{33} \bar{\zeta}_{2}^{2}=\bar{g}(\{\bar{\rho}\}), & \bar{\alpha}_{1} \bar{\alpha}_{3}+\bar{\beta}_{1} \bar{\beta}_{3}+\bar{\gamma}^{33} \bar{\zeta}_{1} \bar{\zeta}_{3}=0, \\
\bar{\alpha}_{3}^{2}+\bar{\beta}_{3}^{2}+\bar{\gamma}^{33} \bar{\zeta}_{3}^{2}=\bar{g}(\{\bar{\rho}\}), & \bar{\alpha}_{2} \bar{\alpha}_{3}+\bar{\beta}_{2} \bar{\beta}_{3}+\bar{\gamma}^{33} \bar{\zeta}_{2} \bar{\zeta}_{3}=0,
\end{aligned}
$$




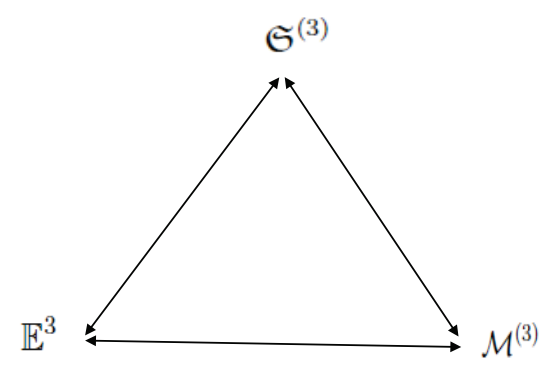

Fig. 1: In this diagram all spaces are homeomorphic to each other, i.e., $\mathbb{E}^{3} \simeq \mathfrak{S}^{(3)} \simeq \mathcal{M}^{(3)}$.

where $\bar{g}(\{\bar{\rho}\})=\breve{g}(\{\bar{\rho}\}+\{\delta \bar{\rho}\}), \quad\{\delta \bar{\rho}\}=\left(\delta \rho^{1}, \delta \rho^{2}, \delta \rho^{3}\right)$. Assuming that $|\delta\{\bar{\rho}\}| \ll 1$, in the equations (11), we can expand the functions in a Taylor series on these small parameters and taking into account the system of equations (9), we get:

$$
\begin{aligned}
\delta \rho^{i}\left\{2\left(\alpha_{1} \alpha_{1 i}+\beta_{1} \beta_{1 i}+\gamma^{33} \zeta_{1} \zeta_{1 i}\right)+\gamma_{, i}^{33} \zeta_{1}^{2}-\breve{g}_{, i}(\{\bar{\rho}\})\right\} & =O\left(|\delta\{\bar{\rho}\}|^{2}\right), \\
\delta \rho^{i}\left\{2\left(\alpha_{2} \alpha_{2 i}+\beta_{2} \beta_{2 i}+\gamma^{33} \zeta_{2} \zeta_{2 i}\right)+\gamma_{, i}^{33} \zeta_{2}^{2}-\breve{g}_{, i}(\{\bar{\rho}\})\right\} & =O\left(|\delta\{\bar{\rho}\}|^{2}\right), \\
\delta \rho^{i}\left\{2\left(\alpha_{3} \alpha_{3 i}+\beta_{3} \beta_{3 i}+\gamma^{33} \zeta_{3} \zeta_{3, i}\right)+\gamma_{, i}^{33} \zeta_{3}^{2}-\breve{g}_{, i}(\{\bar{\rho}\})\right\} & =O\left(|\delta\{\bar{x}\}|^{2}\right), \\
\delta \rho^{i}\left\{\alpha_{1} \alpha_{2 i}+\alpha_{2} \alpha_{1 i}+\beta_{1} \beta_{2 i}+\beta_{2} \beta_{1 i}+\gamma^{33}\left(\zeta_{1} \zeta_{2 i}+\zeta_{2} \zeta_{1 i}\right)+\gamma_{, i}^{33} \zeta_{1} \zeta_{2}\right\} & =O\left(|\delta\{\bar{\rho}\}|^{2}\right), \\
\delta \rho^{i}\left\{\alpha_{1} \alpha_{3 i}+\alpha_{3} \alpha_{1 i}+\beta_{1} \beta_{3 i}+\beta_{3} \beta_{1 i}+\gamma^{33}\left(\zeta_{1} \zeta_{3 i}+\zeta_{3} \zeta_{1 i}\right)+\gamma_{, i}^{33} \zeta_{1} \zeta_{3}\right\} & =O\left(|\delta\{\bar{\rho}\}|^{2}\right), \\
\delta \rho^{i}\left\{\alpha_{2} \alpha_{3, i}+\alpha_{3} \alpha_{2 i}+\beta_{2} \beta_{3 i}+\beta_{3} \beta_{2 i}+\gamma^{33}\left(\zeta_{2} \zeta_{3 i}+\zeta_{3} \zeta_{2 i}\right)+\gamma_{, i}^{33} \zeta_{2} \zeta_{3}\right\} & =O\left(|\delta\{\bar{\rho}\}|^{2}\right),
\end{aligned}
$$

where $i=\overline{1,3}$ and summation is performed by dummy indices.

If we require that the expressions with the same increments be equal to zero, then from (12) one can obtain an underdetermined system of algebraic equations, i.e., 18 equations for finding 27 unknowns variables:

$$
\begin{array}{r}
2\left(\alpha_{1} \alpha_{1 i}+\beta_{1} \beta_{1 i}+\gamma^{33} \zeta_{1} \zeta_{1 i}\right)+\gamma_{, i}^{33} \zeta_{1}^{2}-\breve{g}_{, i}(\{\bar{\rho}\})=0 \\
2\left(\alpha_{2} \alpha_{2 i}+\beta_{2} \beta_{2 i}+\gamma^{33} \zeta_{2} \zeta_{2 i}\right)+\gamma_{, i}^{33} \zeta_{2}^{2}-\breve{g}_{, i}(\{\bar{\rho}\})=0 \\
2\left(\alpha_{3} \alpha_{3 i}+\beta_{3} \beta_{3 i}+\gamma^{33} \zeta_{3} \zeta_{3 i}\right)+\gamma_{, i}^{33} \zeta_{3}^{2}-\breve{g}_{, i}(\{\bar{\rho}\})=0 \\
\alpha_{2} \alpha_{1 i}+\alpha_{1} \alpha_{2 i}+\beta_{2} \beta_{1 i}+\beta_{1} \beta_{2 i}+\gamma^{33}\left(\zeta_{2} \zeta_{1 i}+\zeta_{1} \zeta_{2 i}\right)+\gamma_{, i}^{33} \zeta_{1} \zeta_{2}=0 \\
\alpha_{3} \alpha_{1 i}+\alpha_{1} \alpha_{3 i}+\beta_{3} \beta_{1 i}+\beta_{1} \beta_{3 i}+\gamma^{33}\left(\zeta_{3} \zeta_{1 i}+\zeta_{1} \zeta_{3 i}\right)+\gamma_{, i}^{33} \zeta_{1} \zeta_{3}=0 \\
\alpha_{3} \alpha_{2 i}+\alpha_{2} \alpha_{3 i}+\beta_{3} \beta_{2 i}+\beta_{2} \beta_{3 i}+\gamma^{33}\left(\zeta_{3} \zeta_{2 i}+\zeta_{2} \zeta_{3 i}\right)+\gamma_{, i}^{33} \zeta_{2} \zeta_{3}=0
\end{array}
$$

Recall that the set of coefficients $\{\sigma\}=\left(\sigma_{1}, \ldots, \sigma_{9}\right)=\left[\alpha=\left(\alpha_{1}, \alpha_{2}, \alpha_{3}\right), \beta=\left(\beta_{1}, \beta_{2}, \beta_{3}\right)\right.$, $\left.\zeta=\left(\zeta_{1}, \zeta_{2}, \zeta_{3}\right)\right]$ belongs to the manifold $\mathfrak{S}^{(3)}$.

Now, we can require that the second derivatives be symmetric $\sigma_{i j}=\sigma_{j i}$, where $\{\sigma\}=[\alpha, \beta, \zeta]$ and $i, j=\overline{1,3}$. This, as can be easily seen, allows us to reduce the number of unknown variables and make the system of equations definite, i.e., 18 equations for 18 unknown variables.

The system of equations (13) can be written in canonical form:

$$
\mathbf{A X}=\mathbf{B}, \quad \mathbf{A}=\left(d_{\mu \nu}\right), \quad \mu, \nu=\overline{1,18}
$$

where $\mathbf{A} \in \mathbb{R}^{18 \times 18}$ is the basic matrix of the system, $\mathbf{B} \in \mathbb{R}^{18}$ and $\mathbf{X} \in \mathbb{R}^{18}$ are columns of free terms and system solutions, respectively. Note that, for an arbitrary point $\left\{\bar{\rho}_{i}\right\} \in \mathbb{E}^{3}$, 

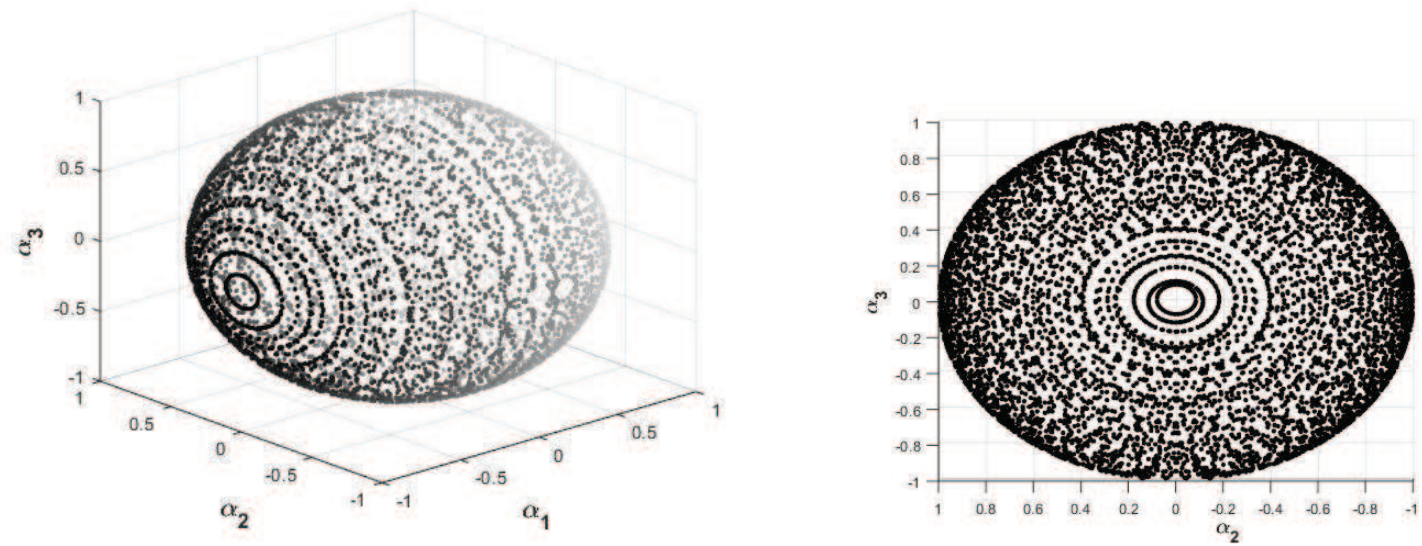

Fig. 2: The form of an oriented manifold generated by a system of equations (9). Note that the calculations of the equations system (9) were performed taking into account the following transformations $\gamma^{33} \zeta_{1} \rightarrow \zeta_{1}, \gamma^{33} \zeta_{2} \rightarrow \zeta_{2}$ and $\gamma^{33} \zeta_{3} \rightarrow \zeta_{3}$. The first figure shows a general view of a manifold in three-dimensional space, which obviously is a sphere with topological features. The second figure shows the projection of a sphere onto a plane $\left(\alpha_{2}, \alpha_{3}\right)$ in the form of a circle, from which one can see a cutting circle in the center. There are obviously six such circular cuts on a sphere.
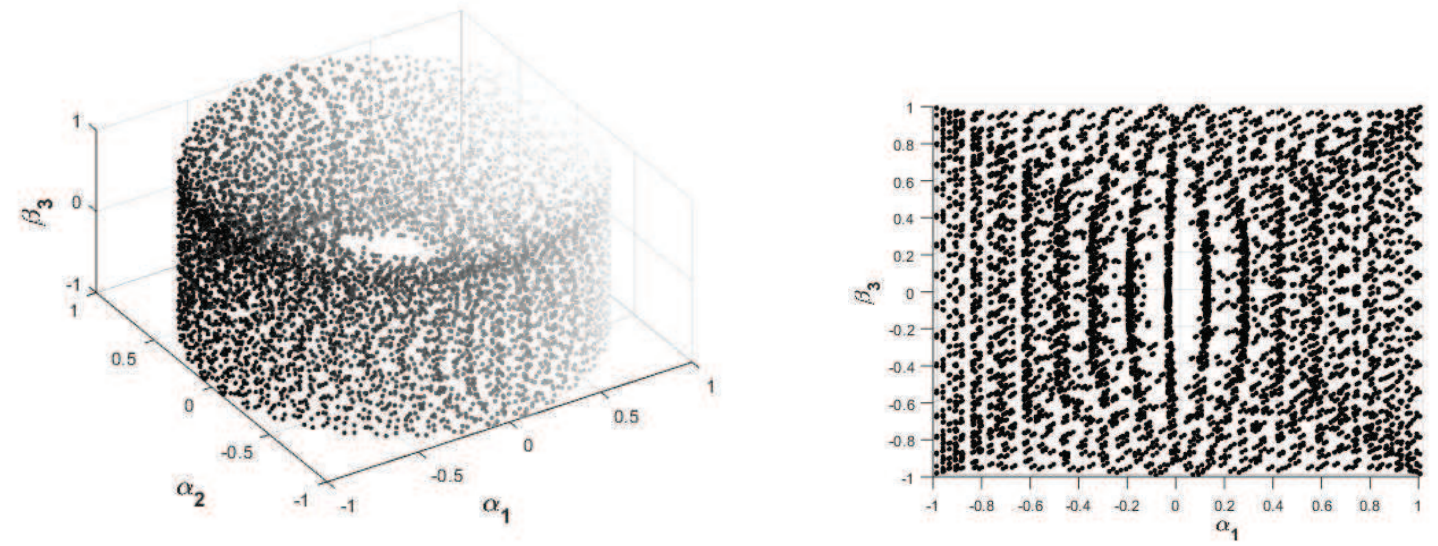

Fig. 3: As can be seen, this manifold also has a topology.
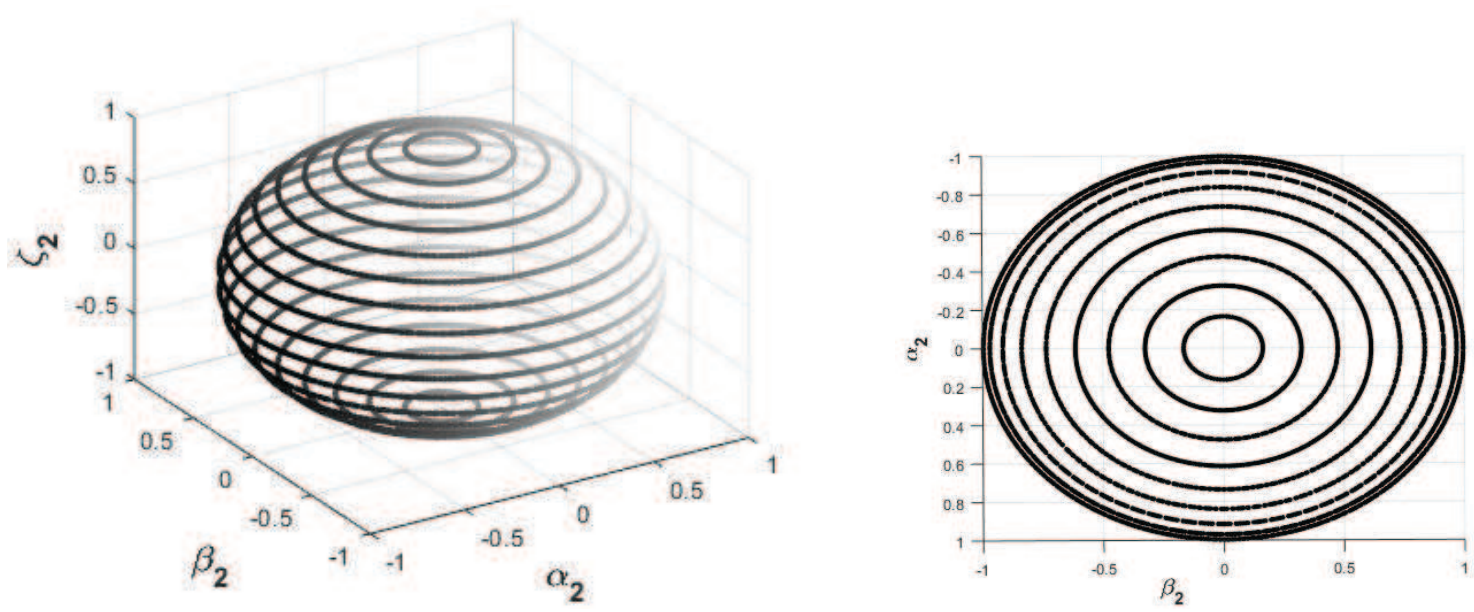

Fig. 4: As can be seen, this manifold also has a topology. 

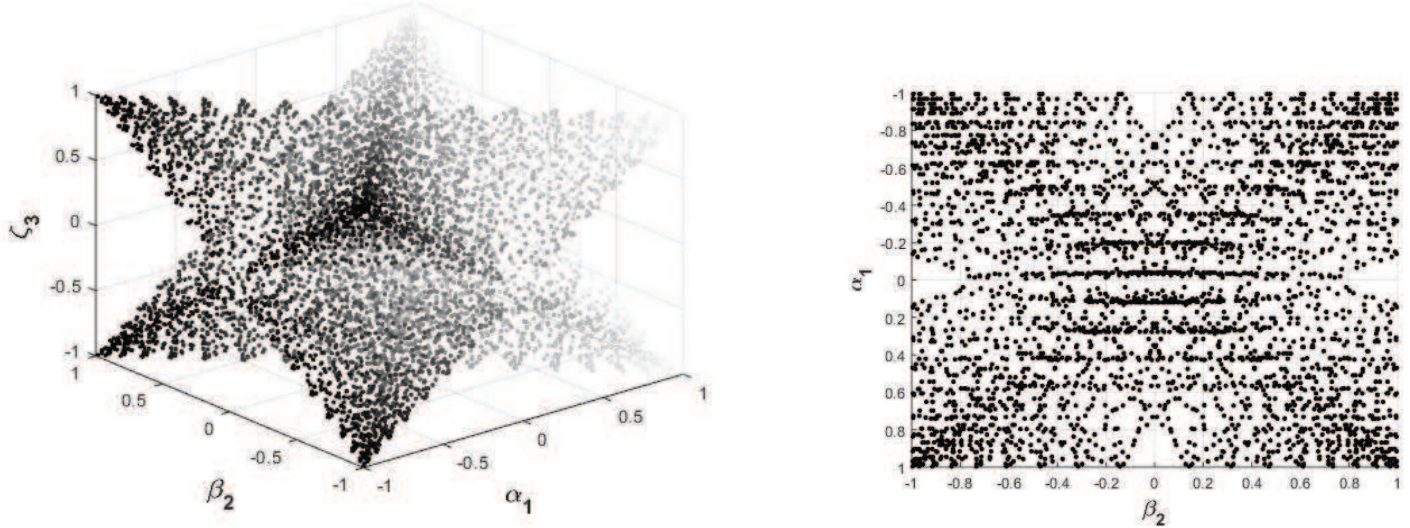

Fig. 5: As can be seen, this manifold also has a topology.

the system of equations (9) generates sets of solutions $\{\sigma\}=[\alpha, \beta, \zeta]$ that continuously fill a region of $\mathbb{E}^{3}$ space, forming $3 D$ manifold $\mathfrak{S}^{(3)}$. As for the system of equations (14), it has a solution if the determinant of the basic matrix $\mathbf{A}$ is nonzero (see Appendix):

$$
\operatorname{det}\left(d_{\mu \nu}\right) \neq 0, \quad \mu, \nu=\overline{1,18}
$$

On the other hand, the algebraic system (14) does not have a solution when $\operatorname{det}\left(d_{\mu \nu}\right)=0$. In this case at each point $\left\{\bar{\rho}_{i}\right\}$ there exists a countable set $\mathfrak{W}$ of coefficients $\{\sigma\}=[\alpha, \beta, \zeta]$ such that $\operatorname{det}\left(d_{\mu \nu}\right)=0$. It is easy to verify that the measure of this set in comparison with the measure of the $\mathfrak{S}^{(3)}$ for which $\operatorname{det}\left(d_{\mu \nu}\right) \neq 0$, is equal to zero, i.e., $\mathfrak{W}=\{0\}$. In other words, for the case under consideration Schwartz's theorem holds, and $\sigma_{\varsigma}$ (where $\varsigma=\overline{1,9}$ ) and $d_{\mu \nu}$ (see (13)) have the sense of the first and second derivatives, respectively.

The same is easily proved for inverse mappings.

Let us consider the open set $\forall G=\cup_{\alpha} G_{\alpha}$, consisting of the union of cards $G_{\alpha}$ arising at continuous mappings $f:\{\bar{\rho}\} \mapsto\{\bar{x}\}$ using algebraic equations (9). Proceeding from the foregoing, it is obvious that the maps can be chosen so that the immediate neighbors have intersections comprising at least one common point, that is a necessary condition for the continuity of the mappings. Using the above arguments, we assert that the atlas $G$ can be widened up to $G \cong \mathcal{M}^{(3)}$.

Now let us discuss the structure of the manifold $\mathcal{M}^{(3)}$. It is easy to see that the independent $\{\sigma\}$ parameters form $9 D$ space $\mathbb{R}^{9}$, in which the system of algebraic equations (9) generates $3 D$ oriented manifolds. These manifolds can be summed up as sets using a certain order by gluing manifolds having common planes. As a result of this gluing, which similar to the the operation of connected sum of topological manifolds, the $3 D$ manifold $\mathcal{M}^{(3)}=\cup_{i} \mathcal{M}_{i}^{(3)}$, is formed. The number of submanifolds $\mathcal{M}_{i}^{(3)}$ can easily be calculated by the formula $C_{n}^{m}=\frac{n !}{m !(n-m) !}$, where $n$ and $m$ denote the dimension of space $\mathbb{R}^{9}$ and the dimension of the manifold $\mathcal{M}_{i}^{(3)}$ immersed into $\mathbb{R}^{9}$, respectively. As the calculations show (see Fig. 2-5), the generated $C_{9}^{3}=84$ topological manifolds can be grouped into four incongruent groups of manifolds. It is also necessary to note that all these varieties are oriented in a 9-dimensional space in the sense that they are well-defined $3 D$ submanifolds.

Thus, all the conditions of the theorem of a homeomorphism between metric spaces $\mathbb{E}^{3}$ and $\mathcal{M}^{(3)}$ are satisfied, and therefore we can say that these spaces are homeomorphic or 
topologically equivalent, i.e., $f: \mathbb{E}^{3} \mapsto \mathcal{M}^{(3)}$.

As for the system of algebraic equations (10), then at each point of the internal space $M_{i}\left(x^{1}, x^{2}, x^{3}\right)_{i} \in \mathcal{M}^{(3)}$, it generates $3 D$ manifold $\mathfrak{R}^{(3)}$ that is a local analogue of the Euler angles and, consequently, $\cup_{i} \mathcal{S}_{M_{i}}^{3} \simeq \mathfrak{R}^{(3)}$. The layer $\mathfrak{R}^{(3)}$, continuously passing through all points of the basis $\mathcal{M}^{(3)}$, fills the subspace $\mathbb{E}^{6}$.

Finally, taking into account the aforesaid, we can conclude that the spaces $\mathbb{E}^{6}$ and $\mathcal{M}$, are homeomorphic too. In addition, the manifold $\mathcal{M}$ can be represented in the form of decomposition $\mathcal{M} \cong \mathcal{M}^{(3)} \times \mathcal{S}_{M_{i}}^{3}$. Proposition 1 is proved.

\section{Conclusion}

As A. Poincaré rightly pointed out, there is no finest geometry, there is a geometry convenient for solving a specific task. Usually, when studying complex dynamical systems, coordinate transformations are used to separate variables and reduce the original system. In particular, by coordinate transformations, the three-body problem, which is a system of 18th order, is reduced to the system of 8 th order. However, as we have shown, it is possible to make the reduction of a dynamical system more complete if we use the curve (Riemannian) geometry. Note that in this case it becomes possible to reveal the hidden symmetries of internal motion and, accordingly, to obtain additional integrals of motion. For a three-body system, replacing the geometry allows us to reduce the problem to the 6th order system. The main difficulty arising at the solution of this problem is the generalization of the well-known Poincaré theorem on a homomorphism between the $3 D$ sphere with unit radius and $3 D$ compact. In this work, the possibility of such a generalization is strictly proved.

\section{Appendix}

As mentioned (see (14)), the vector $\mathbf{X}$ consists of 18 independent components. Its transposed form looks like this:

$$
\mathbf{X}^{T}=\left(\alpha_{11}, \alpha_{12}, \alpha_{13}, \alpha_{22}, \alpha_{23}, \alpha_{33}, \beta_{11}, \beta_{12}, \beta_{13}, \beta_{22}, \beta_{23}, \beta_{33}, \zeta_{11}, \zeta_{12}, \zeta_{13}, \zeta_{22}, \zeta_{23}, \zeta_{33}\right)
$$

Taking into account the form of the vector $\mathbf{X}$, we can write the explicit form of the basic matrix:

$$
\mathbf{A}=\left(\begin{array}{ccc}
d_{1}^{1} & \cdots & d_{1}^{18} \\
\cdots & \cdots & \cdots \\
d_{18}^{1} & \cdots & d_{18}^{18}
\end{array}\right)
$$

where the superscript indicates the column number, while the subscript indicates the line number. As for the explicit form of elements $d_{\mu}^{\nu}=d_{\mu \nu}$, where $\mu, \nu=\overline{1,18}$, then we can find them by multiplying the basic matrix $\mathbf{A}$ with the vector $\mathbf{X}$ (see equation (14)) and comparing with the system of equations (13). In particular, it is easy to verify that these 
terms are equal:

$$
\begin{aligned}
& d_{1}^{1}=d_{2}^{2}=d_{3}^{3}=2 d_{10}^{2}=2 d_{11}^{4}=2 d_{12}^{5}=2 d_{13}^{3}=2 d_{14}^{5}=2 d_{15}^{6}=2 \alpha_{1}, \\
& d_{4}^{2}=d_{5}^{4}=d_{6}^{5}=2 d_{10}^{1}=2 d_{11}^{2}=2 d_{12}^{3}=2 d_{16}^{3}=2 d_{17}^{5}=2 d_{18}^{6}=2 \alpha_{2}, \\
& d_{7}^{3}=d_{8}^{5}=d_{9}^{6}=2 d_{13}^{1}=2 d_{14}^{2}=2 d_{15}^{3}=2 d_{16}^{2}=2 d_{17}^{4}=2 d_{18}^{5}=2 \alpha_{3}, \\
& d_{7}^{1}=d_{8}^{2}=d_{9}^{3}=2 d_{10}^{8}=2 d_{11}^{10}=2 d_{12}^{11}=2 d_{13}^{9}=2 d_{14}^{11}=2 d_{15}^{12}=2 \beta_{1}, \\
& d_{4}^{8}=d_{5}^{10}=d_{11}^{6}=2 d_{10}^{7}=2 d_{11}^{8}=2 d_{12}^{9}=2 d_{16}^{9}=2 d_{17}^{11}=2 d_{18}^{12}=2 \beta_{2}, \\
& d_{7}^{9}=d_{6}^{11}=d_{12}^{6}=2 d_{13}^{7}=2 d_{14}^{8}=2 d_{15}^{9}=2 d_{16}^{8}=2 d_{17}^{10}=2 d_{18}^{11}=2 \beta_{3}, \\
& d_{13}^{1}=d_{14}^{2}=d_{15}^{3}=2 d_{10}^{17}=2 d_{11}^{16}=2 d_{12}^{17}=2 d_{13}^{15}=2 d_{14}^{17}=2 d_{15}^{18}=2 \gamma^{33} \zeta_{1}, \\
& d_{4}^{14}=d_{5}^{16}=d_{17}^{6}=2 d_{10}^{13}=2 d_{11}^{14}=2 d_{12}^{15}=2 d_{13}^{13}=2 d_{14}^{14}=2 d_{15}^{15}=2 \gamma^{33} \zeta_{2}, \\
& d_{7}^{15}=d_{8}^{17}=d_{18}^{9}=2 d_{16}^{14}=2 d_{17}^{16}=2 d_{18}^{17}=2 d_{16}^{15}=2 d_{17}^{17}=2 d_{18}^{18}=2 \gamma^{33} \zeta_{3} .
\end{aligned}
$$

All elements of the matrix (15) missing in (16) are identically zero.

As is known, the algebraic system (13) or (14) does not have a solution in the case when the determinant of the matrix is zero $\operatorname{det}(\mathbf{A})=\operatorname{det}\left(d_{\mu \nu}\right)=0$. A class consisting of sets of coefficients $\{\sigma\}$ for which the determinant is zero, can be countable and the measure, respectively, will be equal to zero $\mathfrak{W}=\{0\}$.

\section{References}

[1] E. A. Ayryan, A. S. Gevorkyan and L. A. Sevastyanova, "On the motion of a three body system on hypersurface of proper energy", Physics of Particles and Nuclei Letters, vol.10, no. 7, pp. 1-8, 2013.

[2] A. S. Gevorkyan, "On reduction of the general three-body Newtonian problem and the curved geometry", Journal of Physics: Conference Series, 496, 012030, 2014.

[3] A. S. Gevorkyan, "On the motion of classical three-body system with consideration of quantum fluctuations", Physics of Atomic Nuclei, vol. 80, no. 2, pp. 358-365, 2017.

[4] A. S. Gevorkyan, "Fundamental irreversibility and times arrow of the classical threebody problem. New approaches and ideas in the study of dynamical systems". arXiv:1706.09827v2[math-ph] 13 Dec 201\%.

[5] A. S. Gevorkyan, "Is the Hamiltonian mechanics and in general classical mechanics reversible?", Book of abstracts, International Conference Dedicated to the 120th Anniversary of Emil Artin, Yerevan, Armenia, May 27-June 2, pp. 58-59, 2018.

Submitted 04.12.2018, accepted 23.04.2019. 


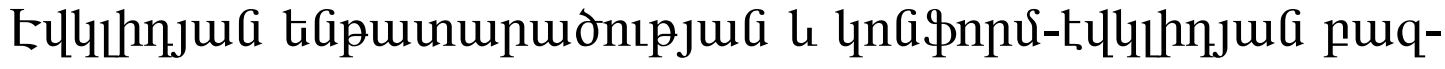

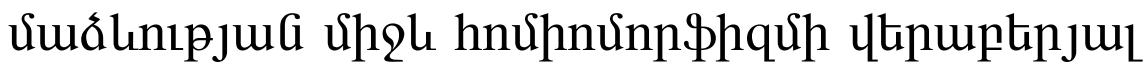

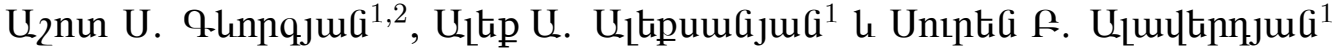

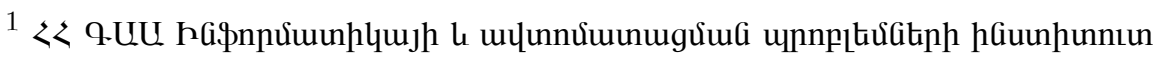

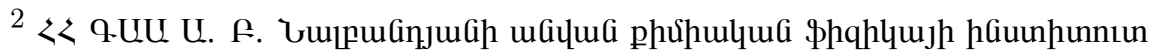 \\ e-mail: gashot@ipia.sci.am
}

\section{Uরuนnนhnน์}

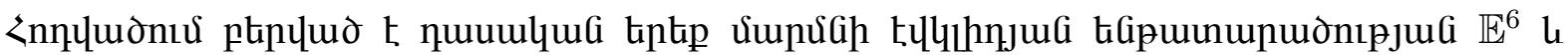

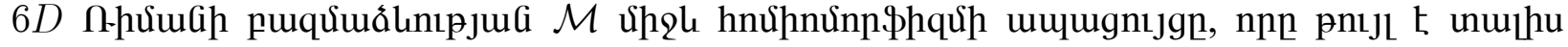

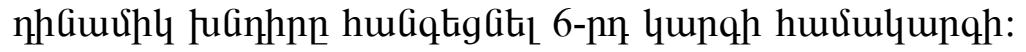

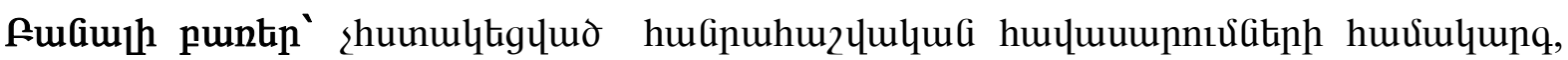

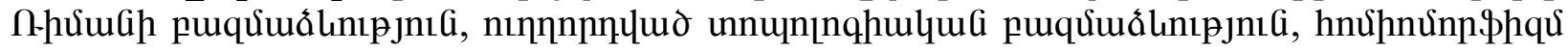
puqưuálınıpjnıGitinh ưh̨h:

\section{О гомеоморфизме меЖАу евклидовым подпространством и конформно-евклидовым многообразием}

\author{
Ашот С. Геворкян ${ }^{1,2}$, Алек А. Алексанян ${ }^{1}$ и Сурен Б. Алавердян ${ }^{1}$ \\ ${ }^{1}$ Институт проблем информатики и автоматизации НАН РА \\ ${ }^{2}$ Институт химической физики имени А. Б. Налбандяна НАН РА \\ e-mail: gashot@ipia.sci.am
}

\begin{abstract}
Аннотация
В статье представлено доказательство гомеоморфизма между евклидовым подпространством $\mathbb{E}^{6}$ классической системы трех тел и $6 D$ римановым многообразием $\mathcal{M}$, что позволяет свести динамическую задачу к системе 6-го порядка.

Ключевые слова: система недоопределенных алгебраических уравнений, риманово многообразие, ориентированное топологическое многообразие, гомеоморфизм между многообразиями.
\end{abstract}

